\title{
Comparison of ultrasound guidance for axillary or subclavian vein catheterization: a randomized controlled non-inferiority trial
}

\author{
B Louart*', G Buzançais, C Roger, L Muller, JY Lefrant \\ From ESICM LIVES 2015 \\ Berlin, Germany. 3-7 October 2015
}

\section{Introduction}

Axillary vein catheterization appears as an interesting alternative to subclavian vein catheterization (SVC) under ultrasound (US) guidance. $\left[{ }^{1}\right]$

\section{Objectives}

The aim of this trial was to compare the two approaches.

\section{Methods}

Randomized non-inferiority single-centre study. All patients admitted in intensive care unit (ICU) or operating room, requiring a central vein catheterization $(\mathrm{CVC})$ without contraindication for SVC, were randomly assigned to subclavian or axillary groups. The primary endpoint was to compare success rate of each approach. The secondary endpoints were: strategy success rates, catheter position and complications. Strategy of CVC consisted in using the allocated approach and switching to the non-allocated approach after two failed punctures.

\section{Results}

122/132 included patients were analysed (60 and 62 in subclavian and axillary group, respectively). The approach success rates for subclavian and axillary sites were $87.7 \%$ and $85.5 \%$, respectively (difference $-2.2 \%$, $90 \%$ CI $[-12.5 \%-8.1 \%]$, non-inferiority $\mathrm{p}=0.18$ ). The subclavian and axillary strategy success rates were $96.5 \%$ and 98.4\%, respectively (difference $-1.9 \%, 90 \%$ CI [-4.9\%-8.7\%], non-inferiority $\mathrm{p}<0.01)$. Thrombogenic catheter positions were 7 (12.3\%) in subclavian group vs. $19(31.7 \%)$ in axillary group $(\mathrm{p}=0.01)$. Complications were comparable in the two groups $(2(3.3 \%)$ vs. $4(6.5 \%), \mathrm{p}=0.68)$.
Table 1 Success rates for subclavian and axillary groups.

\begin{tabular}{llll}
\hline & Subclavian group & Axillary group & $\mathbf{p}$ \\
\hline Success rates (\%, 95Cl) & & & \\
\hline Approach & $88.3[77.4-95.2]$ & $85.5[74.2-93.1]$ & 0.202 \\
\hline Strategy & $96.7[88.5-99.6]$ & $98.4[91.3-99.7]$ & 0.009 \\
\hline First puncture & $66.7[53.3-78.3]$ & $67.7[54.7-79.1]$ & 0.142 \\
\hline
\end{tabular}

\section{Conclusions}

In terms of absolute success rate, axillary is not noninferior to subclavian approach. In terms of strategy success rate, axillary is non-inferior to subclavian approach. After two failed subclavian approaches, changing for axillary approach leads to $98 \%$ success rate. Although associated with more thrombogenic catheter extremity position, axillary approach can be considered as a rescue alternative after subclavian approach failure.

\section{Published: 1 October 2015}

\section{Reference}

1. Sharma A, Bodenham AR, Mallick A: Ultrasound-guided infraclavicular axillary vein cannulation for central venous access. Br J Anaesth 2004, 93:188-92.

doi:10.1186/2197-425X-3-S1-A614

Cite this article as: Louart et al.: Comparison of ultrasound guidance for axillary or subclavian vein catheterization: a randomized controlled non-inferiority trial. Intensive Care Medicine Experimental 2015 3(Suppl 1): A614. 